\title{
On the existence of Einstein oscillators and thermal conductivity in bulk metallic glass
}

\author{
Zhenhua Zhou a) and Ctirad Uher \\ Department of Physics, University of Michigan, Ann Arbor, Michigan 48109 \\ Donghua $\mathrm{Xu}^{\mathrm{b})}$ and $\mathrm{W}$. L. Johnson \\ Keck Laboratory of Engineering Materials, California Institute of Technology, Pasadena, California 91125
}

W. Gannon and M. C. Aronson

Department of Physics, University of Michigan, Ann Arbor, Michigan 48109

(Received 27 January 2006; accepted 19 June 2006; published online 21 July 2006)

\begin{abstract}
Low-temperature specific heat and thermal conductivity of bulk metallic glasses are measured to identify the primary vibrational modes associated with their unique structures. An Einstein-type localized vibrational mode with an Einstein temperature of $112 \mathrm{~K}$ is found in bulk metallic glass $\mathrm{Ni}_{59.5} \mathrm{Nb}_{33.6} \mathrm{Sn}_{6.9}$. This localized vibrational mode causes resonant scattering of phonons and results in the localization of phonons which leaves the phonon hopping conduction the limiting mechanism of thermal transport in bulk metallic glass $\mathrm{Ni}_{59.5} \mathrm{Nb}_{33.6} \mathrm{Sn}_{6.9}$ at high temperature. (c) 2006 American Institute of Physics. [DOI: 10.1063/1.2234281]
\end{abstract}

The low-temperature specific heat and thermal conductivity of rapidly quenched metallic glasses had been the subjects of experimental investigations. ${ }^{1-3}$ Recently, bulk metallic glasses (BMGs) with a thickness of $1 \mathrm{~mm}$ or larger have been found in a number of alloy systems which usually contains three or more elements with large atomic size difference. ${ }^{4,5}$ BMGs differ from the rapidly quenched metallic glasses in the fact that a significantly slower cooling rate is required to get bulk glassy alloys. This is usually due to their strong viscosity-temperature dependence and the formation of polytetrahedral short range ordering (SRO) in undercooled alloy melts during cooling. ${ }^{6}$ As a result, the phonon specific heat $C_{\text {phonon }}$ of metallic glass may not be described by the Debye model only and additional vibrational modes which are related with their unique SRO structures can exist. It is important to clarify the nature of these additional vibration modes since they not only modify the overall vibrational mode distribution but also influence the phonon thermal conductivity $\kappa_{L}$ of BMG. $\kappa_{L}$ is determined by the vibrational excitations and their mutual interactions. In this letter, we report on the existence of Einstein-type oscillators in a typical BMG and their influence on the phonon thermal conductivity.

The metallic glass samples used in this study have the composition $\mathrm{Ni}_{59.5} \mathrm{Nb}_{33.6} \mathrm{Sn}_{6.9}$. Their synthesis and structural characterization were similar to those reported by Choi-Yim et $a .^{7}$ The specific heat measurements were carried out between 1.8 and $154 \mathrm{~K}$ with the heat capacity option of the physical property measurement system (PPMS) from Quantum Design. The relative error is estimated to be within $2 \%$ based on the instrument specifications. The electrical resistivity $(\rho)$ and thermal conductivity $(\kappa)$ were measured from 5 to $300 \mathrm{~K}$ in a cryostat equipped with a copper radiation shield. We employed a longitudinal steady-state method and used $\mathrm{Au}-0.07$ at. \% Fe-Chromel thermocouples. The absolute accuracy of the $\kappa$ measurement is limited to about $\pm 5 \%$

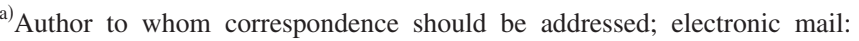
zhenhua.zhou@delphi.com

${ }^{b}$ Present address: Department of Nuclear Engineering, University of California, Berkeley, CA 94720.
}

by the uncertainty in the thermocouple junction separation.

Magnetization measurements show that BMG $\mathrm{Ni}_{59.5} \mathrm{Nb}_{33.6} \mathrm{Sn}_{6.9}$ is paramagnetic from 300 to $1.8 \mathrm{~K}$ with the susceptibility of $1.28 \times 10^{-6} \mathrm{emu} /(\mathrm{g} \mathrm{Oe})$. There is no magnetic contribution to $C_{P}$ at zero magnetic field. Therefore, $C_{P}$ is the sum of two terms: electronic specific heat $C_{\text {electron }}$ and phonon specific heat $C_{\text {phonon }}$. In the temperature range of $33.4 \leqslant T^{2} \leqslant 413.2 \mathrm{~K}^{2}$, we fit the measured $C_{P}$ to an expression $C_{P}(T)=\gamma T+\beta T^{3}$ where the first term represents $C_{\text {electron }}$ and the second term stands for $C_{\text {phonon }}$ based on Debye model. The fitting parameters are $\gamma=1.77 \mathrm{~mJ} \mathrm{~mol}^{-1} \mathrm{~K}^{-2}$ and $\beta=88.0 \mu \mathrm{J} \mathrm{mol}^{-1} \mathrm{~K}^{-4}$. $C_{\text {phonon }}$ is determined by subtracting $C_{\text {electron }}$ from the measured $C_{P}$. Figure 1 shows the phonon specific heat $C_{\text {phonon }}$ as well as $C_{\text {phonon }} / T^{3}$ plotted as a function of temperature in the range of $1.8 \leqslant T \leqslant 154 \mathrm{~K}$. The solid lines in Fig. 1 are obtained by fitting the phonon specific heat with the aid of the Debye model in the form of

$$
\frac{C_{\text {phonon }}(T)}{3 R}=F_{D}\left(\frac{\theta_{D}}{T}\right),
$$

where $R$ is the gas constant, $\theta_{D}$ is the Debye temperature, and $F_{D}\left(\theta_{D} / T\right)$ is the Debye function defined as

$$
F_{D}(x)=3\left(\frac{1}{x}\right)^{3} \int_{0}^{x} \frac{e^{z} z^{4}}{\left(e^{z}-1\right)^{2}} d z
$$

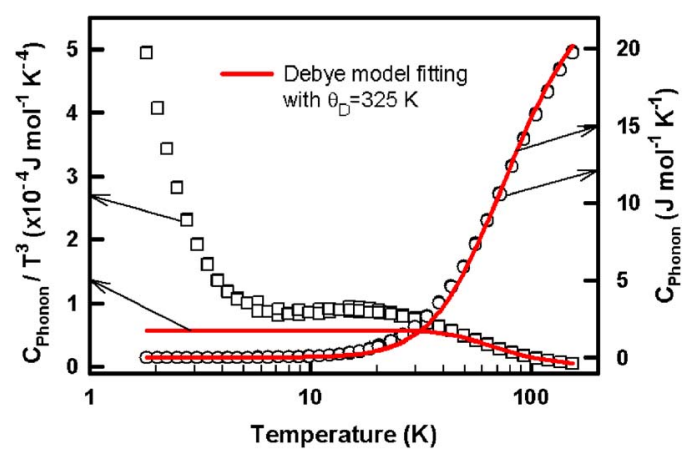

FIG. 1. (Color online) Temperature dependent phonon specific heat of BMG $\mathrm{Ni}_{59.5} \mathrm{Nb}_{33.6} \mathrm{Sn}_{6.9}$. The solid line is a fitting of the specific heat data based on Debye model. 


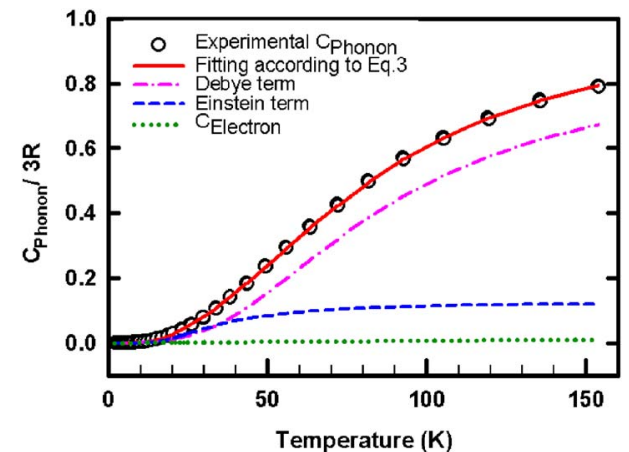

FIG. 2. (Color online) Temperature dependent phonon specific heat. The Debye term and Einstein term are plotted to show their relative weight. The solid line is a fitting curve based on Eq. (3) with parameters described in the text. The dot line shows the electronic specific heat which is a small percentage compared with $C_{\text {phonon }}$.

The fitting gives the Debye temperature $\theta_{D}=325 \mathrm{~K}$. On the $C_{\text {phonon }} / T^{3} \sim T$ plot in Fig. 1, we also note an excess of phonon specific heat over that predicted by the Debye $T^{3}$ model between 5 and $50 \mathrm{~K}$. These results indicate that the Debye model itself cannot explain the experimental phonon specific heat data.

It has been known that localized harmonic vibration modes may exist in amorphous materials. ${ }^{8-11}$ These localized vibration modes will result in an additional Einstein-type vibration, i.e., localized harmonic vibration with a specific frequency. If one assumes that the phonon specific heat of the BMG $\mathrm{Ni}_{59.5} \mathrm{Nb}_{33.6} \mathrm{Sn}_{6.9}$ sample is coming from both the Debye-type and Einstein-type vibration modes, the following expression can be used to model the experimental phonon specific heat:

$$
\frac{C_{\text {phonon }}}{3 R}=n_{D} F_{D}\left(\frac{\theta_{D}}{T}\right)+n_{E} F_{E}\left(\frac{\theta_{E}}{T}\right) .
$$

Here $n_{D}$ and $n_{E}$ are dimensionless Debye-type and Einsteintype vibration strengths, respectively. $\theta_{E}$ is the Einstein temperature and $F_{E}\left(\theta_{E} / T\right)$ is the Einstein function of the following form:

$$
F_{E}(x)=\frac{x^{2} e^{x}}{\left(e^{x}-1\right)^{2}}
$$

Figure 2 shows the total phonon specific heat and the fits according to Eq. (3) in order to determine the relative weight of Debye and Einstein terms. The fits are very good and return the following parameters: $n_{D}=0.873, \theta_{D}=361 \mathrm{~K}, n_{E}$ $=0.127$, and $\theta_{E}=112 \mathrm{~K}$. Recently, it was shown that lowtemperature specific heat indicates the existence of Einstein oscillators with $\theta_{E}$ of $74 \mathrm{~K}$ in BMG $\mathrm{Zr}_{46.75} \mathrm{Ti}_{8.25} \mathrm{Cu}_{7.5} \mathrm{Ni}_{10} \mathrm{Be}_{27.5}$ and $\theta_{E}$ of $88.2 \mathrm{~K}$ in $\mathrm{Cu}_{50} \mathrm{Zr}_{50}$ metallic glass. ${ }^{12,13}$ It should be noted that these fitting parameters of $\theta_{E}$ are not absolute.

Figure 3(a) shows the temperature dependent $\rho$ and $\kappa$ of BMG $\mathrm{Ni}_{59.5} \mathrm{Nb}_{33.6} \mathrm{Sn}_{6.9}$ from 5 to $300 \mathrm{~K}$. $\rho$ is nearly constant in the range of temperature of $5<T<50 \mathrm{~K}$ and then decreases linearly when the temperature increases toward $300 \mathrm{~K}$, i.e., it displays a negative temperature coefficient of resistivity. At temperatures below around $12 \mathrm{~K}, \kappa$ increases rapidly with increasing temperature. This is followed by a weaker temperature dependence that eventually approaches a linear dependence in temperature. The electronic thermal conductivity contribution is estimated using the experimental
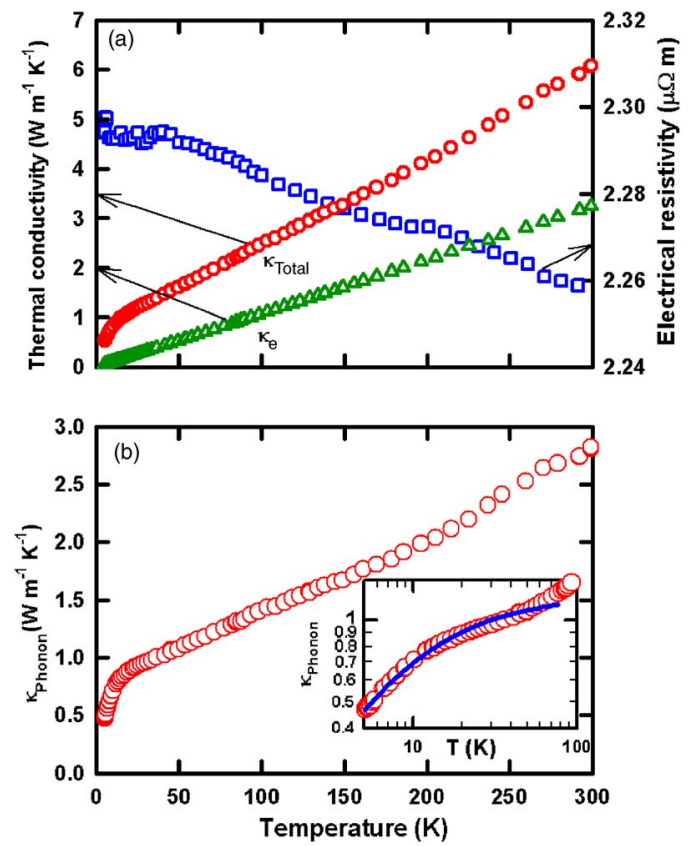

FIG. 3. (Color online) (a) Electrical resistivity $\rho$ and total thermal conductivity $\kappa$, electrical thermal conductivity $\kappa_{e}$, and (b) phonon thermal conductivity $\kappa_{L}$ as a function of temperature for BMG $\mathrm{Ni}_{59.5} \mathrm{Nb}_{33.6} \mathrm{Sn}_{6.9}$. The solid line in the inset of (b) is a fit to the low-temperature phonon thermal conductivity according to Eq. (5) with relaxation time $\tau_{C}$ expressed in Eq. (6) with parameters described in the text.

electrical resistivity and the Wiedemann-Franz law with the ideal Lorenz number, $L_{0}=2.44 \times 10^{-8} \mathrm{~W} \Omega / \mathrm{K}^{2}$.

Figure 3(b) shows the temperature dependence of the phonon thermal conductivity $\kappa_{L}$ of BMG $\mathrm{Ni}_{59.5} \mathrm{Nb}_{33.6} \mathrm{Sn}_{6.9}$. The phonon thermal conductivity was obtained by subtracting the electronic thermal conductivity. The most striking feature of $\kappa_{L}$ is its near-linear dependence on temperature above about $20 \mathrm{~K}$ together with the existence of a weakly temperature dependent plateau observed between 12 and $20 \mathrm{~K}$. This is in sharp contrast with simple crystalline solids whose $\kappa_{L}$ decreases as $1 / T$ due to the dominant phononphonon Umklapp processes. At lower temperatures below $12 \mathrm{~K}, \kappa_{L}$ is proportional to the temperature. Lowtemperature phonon thermal conductivity is usually determined by the scattering from localized two level systems and shows a typical $T^{2}$ relationship. The fact that we observe the linear dependence instead of the $T^{2}$ variation of $\kappa_{L}$ in BMG $\mathrm{Ni}_{59.5} \mathrm{Nb}_{33.6} \mathrm{Sn}_{6.9}$ indicates a participation of additional scattering mechanisms.

Although we have here an amorphous system, the heat transport can be described in terms of phonons ${ }^{14}$ except that Umklapp processes are not allowed due to the lack of longrange periodic structure. ${ }^{15}$ Our specific heat investigations already show the existence of Einstein oscillators in BMG $\mathrm{Ni}_{59.5} \mathrm{Nb}_{33.6} \mathrm{Sn}_{6.9}$. This kind of localized oscillators could originate from the polytetrahedral short-range ordering in the metallic glass system. The localized oscillators themselves do not contribute to the heat conduction. However, they do influence phonon transport through anharmonic interactions. This will introduce an additional term to the total phonon relaxation time.

We analyze the low-temperature phonon thermal conductivity $(5-80 \mathrm{~K})$ of $\mathrm{BMG} \mathrm{Ni}_{59.5} \mathrm{Nb}_{33.6} \mathrm{Sn}_{6.9}$ with the aid of the Debye model. The phonon thermal conductivity is expressed in the following form: 


$$
\kappa_{L}=\frac{k_{B}}{2 \pi^{2} v}\left(\frac{k_{B} T}{\hbar}\right)^{3} \int_{0}^{\theta_{D} / T} \frac{x^{4} e^{x}}{\tau_{C}^{-1}\left(e^{x}-1\right)^{2}} d x .
$$

Here $\kappa_{L}$ is the phonon thermal conductivity, $x=\hbar \omega / k_{B} T$ is a dimensionless quantity, $\omega$ is the phonon frequency, $k_{B}$ is the Boltzmann constant, $\hbar$ is the reduced Planck constant, $\theta_{D}$ is the Debye temperature, $T$ is the absolute temperature, $v$ is the velocity of sound, and $\tau_{C}$ is the total phonon scattering relaxation time. The Debye temperature $\theta_{D}(361 \mathrm{~K})$ is obtained from the specific heat data. The velocity of sound $(3139 \mathrm{~m} / \mathrm{s})$ is estimated from the following relationship: $v$ $=(k / \hbar)\left(6 \pi^{2}\right)^{-1 / 3} \theta_{D} \gamma$, where $\gamma$ is the cubic root of the atomic volume. There are several possible phonon scattering mechanisms such as external boundary scattering, Rayleigh-type point defect scattering, resonant scattering from localized vibrational modes, and electron-phonon interactions. Boundary scattering gives temperature and frequency independent relaxation times and results in a $T^{3}$-type dependence of $\kappa_{L}$ on $T$ at low temperatures. Rayleigh-type scattering will strongly suppress high frequency phonons since the relaxation time is proportional to $\omega^{-4}$. Attempts to fit the $\kappa_{L}$ with the Debye model that includes only boundary scattering and Rayleightype scattering mechanisms could not reproduce the experimental $\kappa_{L}$ over the interval 5 to $80 \mathrm{~K}$. This suggests that there is another phonon scattering mechanism which gives rise to the weak temperature dependence in $\kappa_{L}$. Trying to fit the phonon thermal conductivity with the Debye model taking into account also resonant scattering due to the interaction between acoustical phonons and localized vibrators has not improved the fit significantly. Only when the electronphonon interaction was taken into account was the fit to the experimental data of the phonon thermal conductivity satisfactory. The following processes are assumed to limit the heat conduction of phonons: Rayleigh-type point defect scattering, resonant scattering, and electron-phonon scattering. The phonon scattering relaxation rate $\tau_{C}^{-1}$ can be written as

$$
\tau_{C}^{-1}=A \omega^{4}+\frac{B \omega^{2} T^{2}}{\left(\omega_{E}^{2}-\omega^{2}\right)^{2}+\gamma \omega_{E}^{2} \omega^{2}}+C \omega^{2} .
$$

Here $A, B$, and $C$ are the fitting parameters which are related to point defect scattering, concentration of impurities causing the resonant scattering, and electron-phonon interaction strength, respectively. $\gamma$ is the average deformation potential. The resonant vibration frequency $\omega_{E}$ is determined by $k_{B} \theta_{E} / \eta$ where $\theta_{E}$ is the Einstein temperature of the localized vibrational mode. The optimal fitting parameters are $A$ $=9.53 \times 10^{-41} \mathrm{~s}^{3}, B=2.5 \times 10^{-6} \mathrm{~K}^{-2} \mathrm{~s}^{-1}, C=8.268 \times 10^{-16} \mathrm{~s}$, and $\gamma=0.5$. The fitting curve is shown as the solid line in Fig. $3(b)$.

Turning attention to higher temperatures $(T>20 \mathrm{~K})$, $\kappa_{L}$ increases nearly linearly with the temperature. Such behavior can be explained with the hopping conduction mechanism. ${ }^{16-18}$ Figure 4 shows the phonon mean free path $l$ calculated based on the experimental $\kappa_{L}$ and specific heat using the kinetic theory expression: $l=3 \kappa_{L} /\left(C_{V} v\right)$. The phonon mean free path diminishes rapidly with increasing temperature and reaches a constant value of around $0.9 \mathrm{~nm}$ when $T>\theta_{E}$. It is worthwhile to note that the wavelength of phonon with the frequency of $\omega_{E}$ is around $1 \mathrm{~nm}$ if we take the velocity of sound as $3139 \mathrm{~m} / \mathrm{s}$. It is apparent that phonons with frequency higher than $\omega_{E}$ will not be able to participate in phonon thermal conductivity since they will be localized.

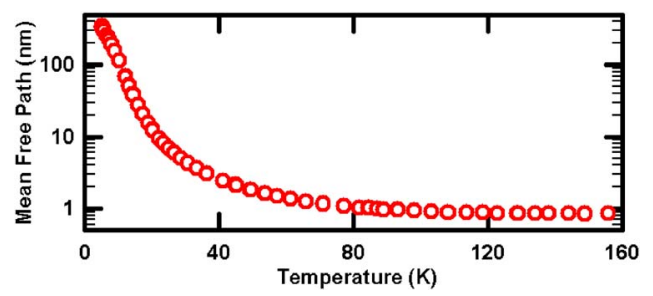

FIG. 4. (Color online) Phonon mean free path as a function of temperature of $\mathrm{BMG} \mathrm{Ni} \mathrm{Ni}_{59.5} \mathrm{Nb}_{33.6} \mathrm{Sn}_{6.9}$ calculated from $C_{\text {phonon }}$ and $\kappa_{L}$.

When $T>\theta_{E}$, the already excited phonons with frequency $\omega<\omega_{E}$ will give a constant contribution to $\kappa_{L}$ since they are already in the Dulong-Petit regime. ${ }^{16,17}$ On the other hand, thermal conduction through hopping between localized phonons can introduce an additional term to the total phonon thermal conductivity. ${ }^{19,20}$ This hopping contribution is proportional to the temperature and the total phonon thermal conductivity will be a linear function of temperature. The linear dependence of $\kappa_{L}$ on temperature shown in Fig. 3(b) validates this viewpoint.

In summary, based on the low-temperature specific heat and thermal conductivity measurements we have shown the existence of Einstein-type localized vibrational mode in bulk metallic glass $\mathrm{Ni}_{59.5} \mathrm{Nb}_{33.6} \mathrm{Sn}_{6.9}$. This localized vibrational mode could originate from the polytetrahedral short-range ordering in the BMG system. It causes resonant scattering of phonons at low-temperature and eventually result in the localization of phonons which leaves the phonon hopping conduction the limiting mechanism of thermal transport in bulk metallic glass.

The work of the two of the authors (W.G. and M.C.A.) is carried out under the auspices of the United States Department of Energy under Grant No. DE-FG02-94ER45526.

${ }^{1}$ J. E. Graebner, B. Golding, R. J. Schutz, F. S. L. Hsu, and H. S. Chen, Phys. Rev. Lett. 39, 1480 (1977).

${ }^{2}$ R. Willnecker, D. M. Herlach, and E. F. Wassermann, Phys. Rev. B 31, 6324 (1985)

${ }^{3}$ J. R. Matey and A. C. Anderson, Phys. Rev. B 16, 3406 (1977).

${ }^{4}$ A. Inoue, A. Takeuchi, and T. Zhang, Metall. Mater. Trans. A 29A, 1779 (1998).

${ }^{5}$ W. L. Johnson and K. Samwer, Phys. Rev. Lett. 95, 195501 (2005).

${ }^{6}$ H. Tanaka, J. Phys.: Condens. Matter 15, L491 (2003).

${ }^{7}$ H. Choi-Yim, D. Xu, and W. L. Johnson, Appl. Phys. Lett. 82, 1030 (2003).

${ }^{8}$ A. D. Caplin, G. Grüner, and J. B. Dunlop, Phys. Rev. Lett. 30, 1138 (1973).

${ }^{9}$ M. Foret, E. Courtens, R. Vacher, and J. Suck, Phys. Rev. Lett. 77, 3831 (1996).

${ }^{10}$ S. Burgess and I. W. Shepherd, Chem. Phys. Lett. 50, 112 (1977).

${ }^{11}$ C. L. Choy, G. L. Salinger, and Y. C. Chiang, J. Appl. Phys. 41, 597 (1970).

${ }^{12}$ M. B. Tang, H. Y. Bai, M. X. Pan, D. Q. Zhao, and W. H. Wang, Appl. Phys. Lett. 86, 021910 (2005).

${ }^{13}$ M. B. Tang, H. Y. Bai, and W. H. Wang, Phys. Rev. B 72, 012202 (2005).

${ }^{14} \mathrm{P}$. G. Klemens, in Non-Crystalline Solids, edited by V. D. Frechette (Wiley, New York, 1960), p. 508.

${ }^{15}$ R. O. Pohl, in Transport and Relaxation in Random Materials, edited by J. Klafter, R. J. Rubin, and M. F. Shlesinger (World Scientific, Philadelphia, 1986), p. 1.

${ }^{16}$ S. Alexander, O. Entin-Wohlman, and R. Orbach, Phys. Rev. B 34, 2726 (1986).

${ }^{17}$ R. Orbach, J. Non-Cryst. Solids 164-166, 917 (1993).

${ }^{18}$ A. Jagannathan and R. Orbach, Phys. Rev. B 41, 3153 (1990).

${ }^{19}$ A. Jagannathan, R. Orbach, and O. Entin-Wohlman, Phys. Rev. B 39, 13465 (1989).

${ }^{20}$ H. Boettger and Th. Damker, Phys. Rev. B 50, 12509 (1994); 59, 8626 (1999). 